\title{
Effet du type de bouture de la palme fourragère (Opuntia ficus-indica (L.) Mill) sur la capacité de reprise et le développement de la plante sous conditions pluviales au Burkina Faso
}

\author{
M. F. OBULBIGA ${ }^{1 *}$, C. A. T. GAVA ${ }^{2}$, V. BOUGOUMA ${ }^{3}$, H. O. SANON $^{1}$, D. KOCTY ${ }^{4}$, \\ D. $\mathrm{KIEMDE}^{3}$, A. COULIBALY ${ }^{4}$ et M. DIALLO ${ }^{4}$ \\ ${ }^{I}$ Département Productions Animales. Institut de l'Environnement et de Recherches Agricoles (INERA) 04 B.P. \\ 8647 Ouagadougou 04, Burkina Faso. \\ ${ }^{2}$ Entreprise Brésilienne de Recherche Agro-pastorale (EMBRAPA). Parque Estaçao Biologica. W3 Norte. Zip \\ Code : 70770-901 Brasília, Brésil. \\ ${ }^{3}$ Université Nazi Boni de Bobo-Dioulasso 01 B.P. 1091 Bobo-Dioulasso 01, Burkina Faso. \\ ${ }^{4}$ Centre National de Multiplication des Animaux Performants, Ministère des Ressources Animales et \\ Halieutiques (MRAH/CMAP) 03 BP 7026 Ouagadougou 03, Burkina Faso. \\ "Auteur correspondant ; E-mail : obulferdi@yahoo.fr
}

\section{RESUME}

Au Burkina Faso, pays sahélien, le problème alimentaire des ruminants, en saison sèche, est préoccupant, en raison de l'état pailleux et sans valeur nutritive de la strate herbacée des parcours naturels. L'amélioration des ressources fourragères est devenue un impératif pour faire face à la diminution quantitative et qualitative de ces parcours due entre autre à la péjoration climatique persistante. Ainsi, l'introduction d'espèces fourragères résistantes bien aux conditions climatiques arides et semi-arides pourrait d'obtenir du fourrage de bonne qualité pour l'alimentation des animaux surtout en saison sèche. L'objectif de l'étude de base est d'évaluer l'effet du type de bouture de l'espèce Opuntia ficus-indica sur la capacité de reprise, l'état de survie et de développement végétatif de la plante sous conditions pluviales en zone nord-soudanienne du Burkina Faso. Les boutures utilisées sont constituées de raquettes sectionnées en trois (03) parties et de raquettes entières âgées de trois (03) mois et récoltées dans un jardin agrostologique sur une population de cent soixante-douze (172) pieds de l'espèce. Le dispositif expérimental a consisté en la plantation des quatre (04) types de bouture : la partie basale $(\mathrm{Pb})$, la partie médiane $(\mathrm{Pm})$, la partie apicale $(\mathrm{Pa})$ et la raquette entière $(\mathrm{Re})$ dans les pots remplis de substrat constitué de un tiers (1/3) de fumier sous forme de poudrette de parc et des deux tiers $2 / 3$ de terreau. La plus grande capacité de reprise des boutures au bout de trois (03) mois (82\%) est obtenue avec la bouture partie basale $(\mathrm{Pb})$. Les boutures partie médiane et raquette entière ont donné la valeur la plus faible $(60 \%)$. Le taux moyen minimal de survie (53\%) est obtenu au niveau de la bouture raquette entière (Re). Ces résultats sur la multiplication végétative de l'espèce Opuntia ficus-indica montrent que la section de la raquette en deux parties pour la production des plants en pots avant leur transplantation en plein champ demeure la technique la mieux indiquée pour la production des plants en grande quantité sous conditions pluviales.

(C) 2018 International Formulae Group. All rights reserved.

Mots clés : Bouture, Palme fourragère, Opuntia ficus-indica, raquette, Burkina Faso. 


\title{
Effect of the type of cutting of the fodder palm (Opuntia ficus-indica (L.) Mill) on the recovery capacity and the development of the plant under rain conditions in Burkina Faso
}

\begin{abstract}
In Burkina Faso, a Sahelian country, the feeding problem of ruminants in the dry season is worrying, due to the strawy state and lack of nutritional value of the herbaceous layer of rangeland. Improvement of fodder resources has become an imperative to cope with the quantitative and qualitative decline of these rangelands due, among other things, to persistent climatic deterioration. Thus, the introduction of forage species that are resistant to arid and semi-arid climatic conditions could provide good quality forage for animal feeding, especially in the dry season. The objective of the basic study is to evaluate the effect of the type of cutting of the species Opuntia ficus-indica on the recovery capacity, the state of survival and vegetative development of the plant under rainy conditions in the North Sudanese zone of Burkina Faso. The cuttings used consist of rackets cut in three (03) parts and entire racket of three (03) months age, and harvested in an agrostological garden on a population of one hundred and seventy-two (172) feet of the species. The experimental design consisted in planting the four (04) types of cutting: the basal part $(\mathrm{Pb})$, the middle part $(\mathrm{Pm})$, the apical part $(\mathrm{Pa})$ and the entire racket $(\mathrm{Re})$ in the pots filled with substrate consisting of one-third $(1 / 3)$ of manure in the form of park powders and two-thirds $2 / 3$ of potting soil. The greatest recovery capacity of the cuttings after three $(03)$ months $(82 \%)$ is obtained with the basal part $(\mathrm{Pb})$ cuttings. Mid-cuttings and entire rackets gave the lowest value $(60 \%)$. The average minimum survival rate $(53 \%)$ is obtained with the entire racket cuttings (Re). These results on the vegetative propagation of the species Opuntia ficus-indica show that the section in two-part of racket for the production of potted plants before their transplantation in the open field remains the most appropriate technique for the production of large quantity of plants under rain conditions.

(C) 2018 International Formulae Group. All rights reserved.
\end{abstract}

Keywords: Cuttings, Fodder palm, Opuntia ficus-indica, racket, Burkina Faso.

\section{INTRODUCTION}

$\mathrm{Au}$ cours des dernières décennies, l'élevage au Burkina Faso a été marqué par un accroissement important des effectifs du cheptel des ruminants (bovins, ovins, caprins). A titre d'exemple, entre 2004 et 2012, l'effectif global de ce cheptel est passé de 24 699000 à 30577000 têtes (MRA/DGPSE, 2013), soit un accroissement de près de $24 \%$. Ce qui a entrâné, sans doute, une pression supplémentaire sur les ressources pastorales et a rendu la situation alimentaire plus précaire pour les animaux. Certes, des travaux de recherche ont été entreprises dans le sens de trouver des solutions à ces contraintes alimentaires des ruminants au Burkina Faso (Bonogo, 2005 ; Sanon, 2007 ; Obulbiga et al., 2007 ; Kabore- Zoungrana et al., 2008 ; Toutain et al., 2009; Klein et al., 2014). Toutefois, la plupart d'entre eux se sont intéressés aux espèces locales, en particulier à la composante herbacée dont la productivité et la contribution à la satisfaction des besoins alimentaires des animaux, reste très fortement tributaire de la pluviosité de l'année.

Le recours à des espèces fourragères pouvant fournir au bétail un fourrage de soudure, en particulier dans les zones sahélienne et nord-soudanienne en saison sèche, s'avère donc nécessaire pour soutenir durablement les productions animales. Opuntia ficus-indica (palme fourragère ou figuier de Barbarie), une plante xérophyte, succulente dont les raquettes ont une grande capacité de rétention d'eau lui conférant une faculté d'adaptation aux régions arides et semi-arides. En effet, ces raquettes ou cladodes assurent la fonction chlorophyllienne et sont recouvertes d'une cuticule cireuse (la cutine) qui limite la transpiration et les protège contre les prédateurs (Neffar, 2012). Elle parait alors une espèce fourragère bien indiquée pour la zone aride et semi-aride du Burkina Faso. En effet, les raquettes 
distribuées à l'animal en complément alimentaire à un autre fourrage ou à un concentré réduisent ainsi considérablement les besoins de celui-ci en eau atteignant $80 \%$ (HCDS, 2002 ; Arba, 2009 ; EMBRAPA, 2014). Aussi, l'espèce, en raison de son appareil racinaire étendu, protège le sol contre l'érosion et également en plantation pourrait être une stratégie prometteuse de conservation des espaces marginaux qui ont perdu toute vocation agropastorale (Neffar et al., 2011).

L'introduction de l'espèce Opuntia ficus-indica au Burkina Faso a eu lieu dans le cadre de l'exécution de l'axe production fourragère du projet de Renforcement de l'Elevage Laitier au Burkina Faso, BRA/04/044S455, projet de coopération technique entre la République Fédérative du Brésil et le Burkina Faso (CMAP, 2015). Les observations préliminaires du comportement de l'espèce introduite nécessitent une évaluation des effets des facteurs de production sur son développement morphologique et sa productivité dans les conditions agro-climatiques du pays. La présente étude vise donc à étudier la technique de production appropriée de plants de l'espèce par bouturage en saison de pluies en zone nord-soudanienne du Burkina Faso afin de mettre au point une méthode de multiplication des plants au profit des agropasteurs.

\section{MATERIELS ET METHODES \\ Site d'étude}

L'étude est réalisée dans la station expérimentale d'élevage de Loumbila située au Nord Est et à $25 \mathrm{~km}$ de Ouagadougou, capitale du Burkina Faso. Ce site d'étude relève du plateau central ou bouclier africain correspondant à l'affleurement du socle et dont la majeure partie est sous forme de pénéplaine de 250 à $300 \mathrm{~m}$ d'altitude. Les sols dominants sont, par ordre d'importance, les sols ferrugineux lessivés peu évolués sur matériaux gravillonnaires, les sols hydromorphes sur matériaux argileux et les lithosols.

Le climat qui y sévit est du type nordsoudanien avec une pluviosité moyenne annuelle comprise entre 600 et $900 \mathrm{~mm}$
(Dembélé, 2010 ; Obulbiga et al., 2014). La saison de pluies dure 4 à 5 mois (juin octobre). Les précipitations connaissent une variabilité interannuelle illustrée par la Figure 1. La moyenne pluviométrie de 1984 - 2012 est de 739,1 mm par an correspondant à un nombre moyen de pluies de soixante-sept (67) jours.

La température moyenne annuelle est de $28,8{ }^{\circ} \mathrm{C}$ avec un maximum de $40{ }^{\circ} \mathrm{C}$ et un minimum de $16{ }^{\circ} \mathrm{C}$. L'humidité relative minimale est de $19 \%$ en janvier et le maximum $67 \%$ en août.

\section{Matériel végétal}

L'espèce Opuntia ficus-indica utilisée dans le cadre de cette étude est un cactus appartenant à la famille des cactacées et à la sous-famille des opuntiatiodées (Arba, 2009). Elle relève du genre Opuntia qui est subdivisé en quatre sous-genres. Les deux sous-genres sont le sous-genre Cylindropuntia comprenant les espèces à tiges cylindriques et le sousgenre Platyopuntia représentant les espèces ayant des raquettes (cladodes) et auquel appartient l'espèce Opuntia ficus-indica ou palme fourragère. Le choix est alors porté sur cette espèce en raison du fait de la possibilité de procéder à sa multiplication par bouturage des raquettes et qu'elle peut servir d'aliment pour l'homme (fruits) et l'animal (raquettes) à la fois (HCDS, 2002). Aussi, elle résiste bien aux conditions climatiques arides et semiarides correspondant à la zone sahélienne et nord-soudanienne du Burkina Faso. Les boutures utilisées sont constituées de morceaux de raquettes adultes de même âge récoltées dans le jardin agrostologique de la station expérimentale d'élevage de Loumbila sur une population de 172 pieds de l'espèce.

Un sécateur a été utilisé pour couper la raquette attachée de la plante et un couteau de cuisine pour son découpage en trois (03) morceaux.

Le sol constitué et mis dans les pots de culture en sachets plastiques en plolyéthylène comportait un tiers $(1 / 3)$ de fumier sous forme de poudrette de parc et des deux tiers $(2 / 3)$ de terreau. 


\section{Méthodes}

Le dispositif expérimental a consisté à la récolte des raquettes adultes de même âge (60 jours) suivie de leur section en morceaux à servir de boutures à planter sur le substrat composé d'un tiers $(1 / 3)$ de fumier et de deux tiers $(2 / 3)$ de terreau. Trois (03) types de bouture ont été obtenus par la découpe de la raquette en trois (3) morceaux: la partie basale $(\mathrm{Pb})$, la partie médiane $(\mathrm{Pm})$ et la partie apicale (Pa). Il s'agit d'un dispositif expérimental en randomisation totale comparant donc quatre (04) types de bouture en trois (03) répétitions ou blocs suivants :

1. un traitement constitué de la plantation de la bouture, partie basale $(\mathrm{Pb})$;

2. un traitement constitué de la plantation de la bouture, partie médiane (Pm) ;

3. un traitement constitué de la plantation de la bouture, partie apicale $(\mathrm{Pa})$;

4. un traitement constitué de la plantation de la raquette entière.

Chaque traitement conduit comportait vingt (20) pots.

\section{Mise en place et entretien des plants dans les pots}

Les raquettes récoltées avaient une longueur moyenne de $21,2 \mathrm{~cm}$ et la largeur $12,4 \mathrm{~cm}$. Les raquettes entières et celles découpées ont toutes été soumises à un préfanage durant quatre (04) jours à l'ombre pour être cicatrisées avant leur mise en pots. A la mise en place des boutures dans les pots, l'orientation Nord Sud a été appliquée pour toutes ces boutures afin de bien assurer leur ensoleillement. Aucune fumure n'a été appliquée durant la conduite de l'essai qui s'est étalée du 5 juillet au 5 octobre 2014. Le désherbage des pots s'est effectué à chaque fois de besoin.

\section{Paramètres agronomiques mesurés}

Les paramètres suivants ont été hebdomadairement mesurés :

1. le nombre de boutures avec présence de bourgeons par type de traitement ;

2. le nombre de boutures mortes et de celles vivantes par type de traitement ;

3. la hauteur et largeur de la raquette primaire et de la raquette secondaire des boutures vivantes par type de traitement trois (3) mois après la plantation en pots.

\section{Analyse statistique}

Les données ont été enregistrées à l'aide du tableur Excel et l'analyse statistique effectuée avec le logiciel SAS System version 9.1.3. Le test de Newman Keuls a été utilisé pour comparer les moyennes au seuil de 5\%.

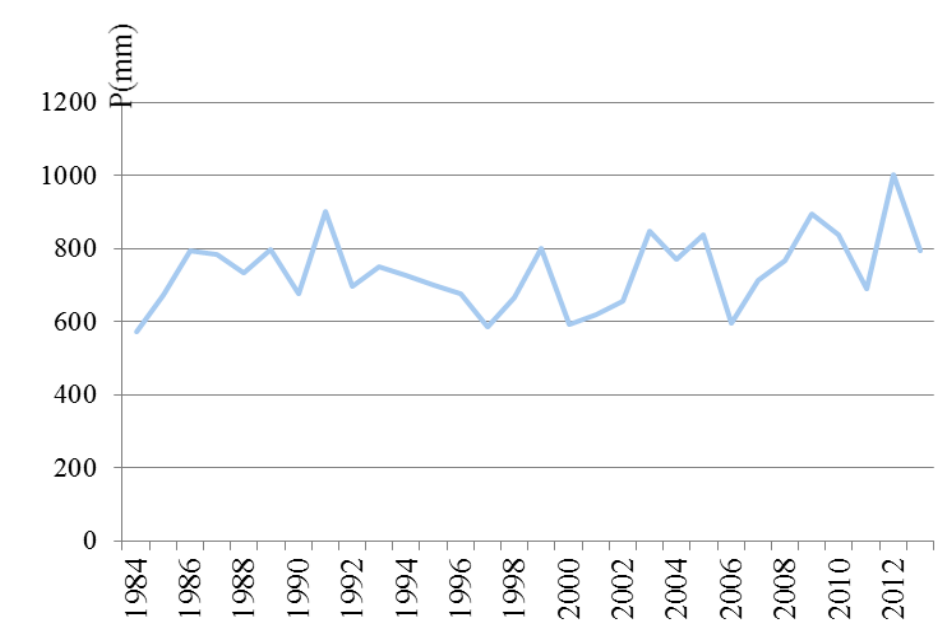

Figure 1 : Pluviométrie de la station expérimentale d'élevage de Loumbila de 1984 à 2012. (Source : Météorologie Nationale, Ouagadougou 2014). 


\section{RESULTATS}

\section{Capacité de reprise des boutures}

L'observation de la capacité de reprise de la plante (Figure 2) montre que la bouture, partie basale de la raquette accuse la plus forte capacité de reprise avec le taux atteignant $88 \%$ dès 28 jours après la mise en pots. A ce stade végétatif, elle est suivie de la bouture, partie apicale et de la bouture, raquette entière accusant respectivement 68 et $67 \%$ de capacité de reprise. Entre le $28^{\text {ème }}$ et $35^{\text {ème }}$ jour, il est observé une légère baisse de cette capacité pour tous les traitements à l'exception de la bouture de la partie médiane qui passe de 38 à $60 \%$. Au terme de trois (03) mois d'observations, la valeur moyenne la plus élevée est obtenue également avec la bouture, partie basale (82\%) suivie de la bouture, partie apicale (63\%.). Les boutures, partie médiane et raquette entière restent égales avec un taux de $60 \%$.

\section{Etat de survie des plants après trois mois de plantation}

Trois (3) mois après la mise en pots des boutures, le traitement, partie basale accuse toujours le taux de survie le plus élevé à savoir $82 \%$, suivie du traitement, partie apicale avec $67 \%$ (Figure 3). Par contre, le taux de survie le plus faible est enregistré au niveau du traitement, raquette entière $(53 \%)$. Toutefois, il convient de souligner que tous les quatre (04) traitements ont un taux de survie supérieur à $50 \%$ après les trois $(03)$ mois de mise en pots des boutures.

\section{Etat de développement végétatif des raquettes émises}

Les dimensions des nouvelles raquettes émises sont consignées dans le Tableau 1. Au niveau des raquettes primaires, la bouture raquette entière accuse la longueur et la largeur les plus élevées qui sont respectivement $22,02 \mathrm{~cm}$ et $13,37 \mathrm{~cm}$. Les valeurs les plus faibles sont observées au niveau du traitement, partie basale avec une longueur moyenne de $19,05 \mathrm{~cm}$ contre une largeur moyenne de $6,73 \mathrm{~cm}$. Il n'a pas été observé une différence significative entre les quatre (4) traitements au seuil de 5\% pour la longueur de la raquette primaire et ainsi de même pour la largeur. Pour ce qui est des raquettes secondaires, la bouture raquette entière donne également la longueur et la largeur maximales à savoir $16,93 \mathrm{~cm}$ et 10,33 $\mathrm{cm}$. Toutefois, aucune différence significative n'a été observée entre les quatre traitements au seuil de $5 \%$ pour chacune de deux variables. Pour les quatre (4) traitements, la longueur moyenne de la raquette primaire âgée de trois (3) mois varie de 19,05 à 22,02 $\mathrm{cm}$ avec une largeur moyenne comprise entre 6,73 et $13,37 \mathrm{~cm}$, soit un rapport Largeur/Longueur variant de 0,35 à 0,61 .

Tableau 1: Dimensions des nouvelles raquettes émises selon le type de bouture.

\begin{tabular}{|c|c|c|c|c|c|}
\hline $\begin{array}{l}\text { Types de } \\
\text { bouture }\end{array}$ & $\begin{array}{c}\text { Rang de la } \\
\text { raquette }\end{array}$ & $\begin{array}{l}\text { Longueur } \\
\text { (cm) }\end{array}$ & $\begin{array}{l}\text { Types de } \\
\text { bouture }\end{array}$ & $\begin{array}{c}\text { Rang de la } \\
\text { raquette }\end{array}$ & Largeur (cm) \\
\hline $\operatorname{Re}$ & $\begin{array}{l}\text { Raquette } \\
\text { primaire }\end{array}$ & $22,02 \pm 1,67 \mathrm{a}$ & $\mathrm{Re}$ & $\begin{array}{l}\text { Raquette } \\
\text { primaire }\end{array}$ & $13,37 \pm 1,10 \mathrm{c}$ \\
\hline $\mathrm{Pm}$ & $\begin{array}{l}\text { Raquette } \\
\text { primaire }\end{array}$ & $20,65 \pm 0,18 \mathrm{a}$ & $\mathrm{Pa}$ & $\begin{array}{l}\text { Raquette } \\
\text { primaire }\end{array}$ & $7,35 \pm 2,07 \mathrm{c}$ \\
\hline $\mathrm{Pa}$ & $\begin{array}{l}\text { Raquette } \\
\text { primaire }\end{array}$ & $20,40 \pm 1,95 \mathrm{a}$ & $\mathrm{Pm}$ & $\begin{array}{l}\text { Raquette } \\
\text { primaire }\end{array}$ & $7,42 \pm 1,56 c$ \\
\hline $\mathrm{Pb}$ & $\begin{array}{l}\text { Raquette } \\
\text { primaire }\end{array}$ & $19,05 \pm 2,81 \mathrm{a}$ & $\mathrm{Pb}$ & $\begin{array}{l}\text { Raquette } \\
\text { primaire }\end{array}$ & $6,73 \pm 0,67 \mathrm{c}$ \\
\hline $\operatorname{Re}$ & $\begin{array}{l}\text { Raquette } \\
\text { secondaire }\end{array}$ & $16,93 \pm 0,92 b$ & $\operatorname{Re}$ & $\begin{array}{l}\text { Raquette } \\
\text { secondaire }\end{array}$ & $10,33 \pm 1,11 d$ \\
\hline Pm & $\begin{array}{c}\text { Raquette } \\
\text { secondaire }\end{array}$ & $16,05 \pm 0,61 b$ & $\mathrm{~Pa}$ & $\begin{array}{c}\text { Raquette } \\
\text { secondaire }\end{array}$ & $7,35 \pm 2,07 d$ \\
\hline $\mathrm{Pb}$ & $\begin{array}{l}\text { Raquette } \\
\text { secondaire }\end{array}$ & $14,37 \pm 1,64 b$ & $\mathrm{Pm}$ & $\begin{array}{l}\text { Raquette } \\
\text { secondaire }\end{array}$ & $6,40 \pm 0,98 \mathrm{~d}$ \\
\hline $\mathrm{Pa}$ & $\begin{array}{l}\text { Raquette } \\
\text { secondaire }\end{array}$ & $12,32 \pm 1,32 b$ & $\mathrm{~Pb}$ & $\begin{array}{l}\text { Raquette } \\
\text { secondaire }\end{array}$ & $6,03 \pm 0,50 \mathrm{~d}$ \\
\hline
\end{tabular}

$\mathrm{Pa}=\mathrm{Partie}$ apicale, $\mathrm{Pm}=$ Partie médiane, $\mathrm{Pb}=\mathrm{Partie}$ basale et $\mathrm{Re}=$ Raquette entière.

Les moyennes affectées d'une même lettre ne diffèrent pas significativement au niveau de $5 \%$. 


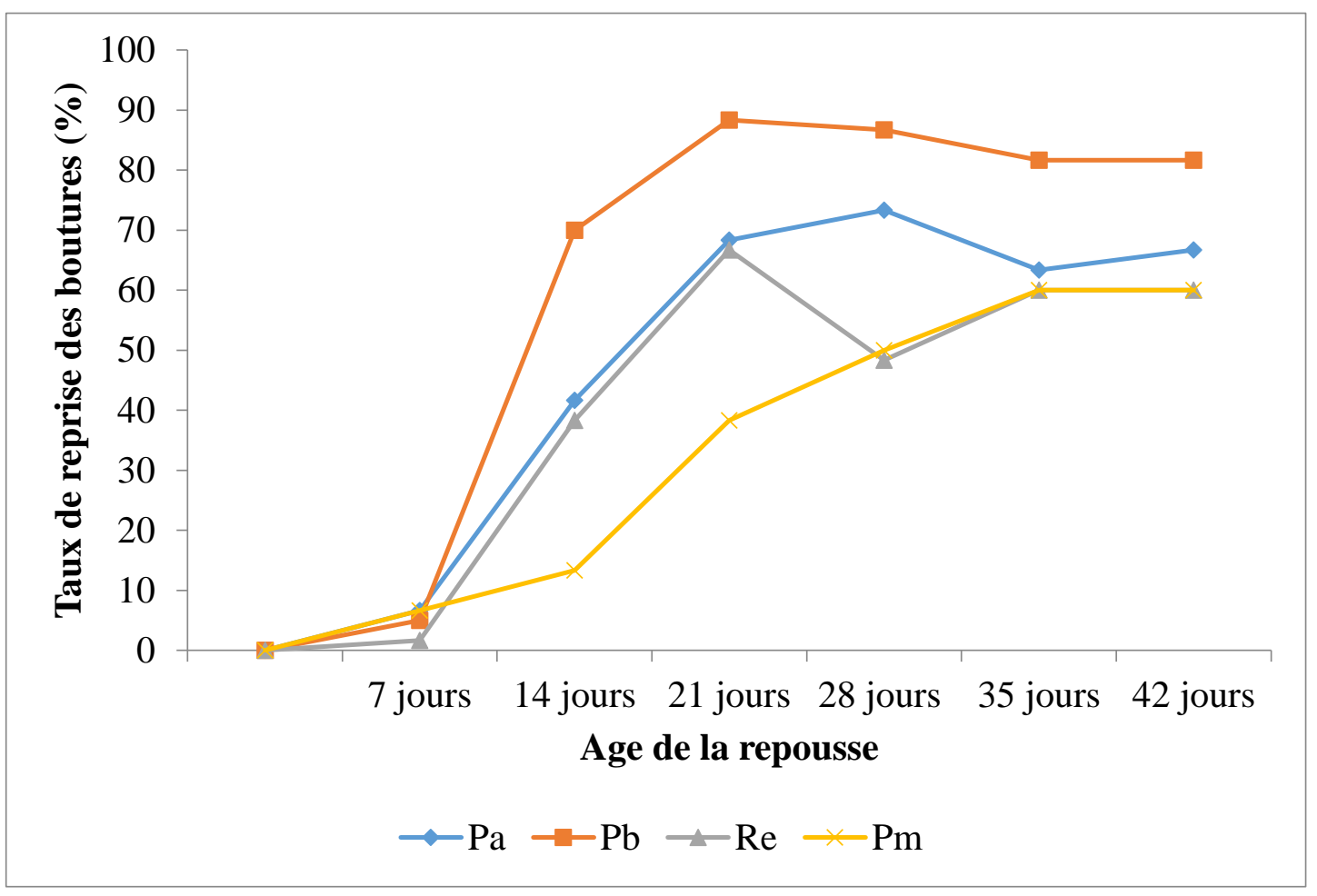

$\mathrm{Pa}=\mathrm{Partie}$ apicale, $\mathrm{Pm}=$ Partie médiane, $\mathrm{Pb}=$ Partie basale et $\mathrm{Re}=$ Raquette entière.

Figure 2: Vitesse de reprise des boutures en fonction du type de bouture.

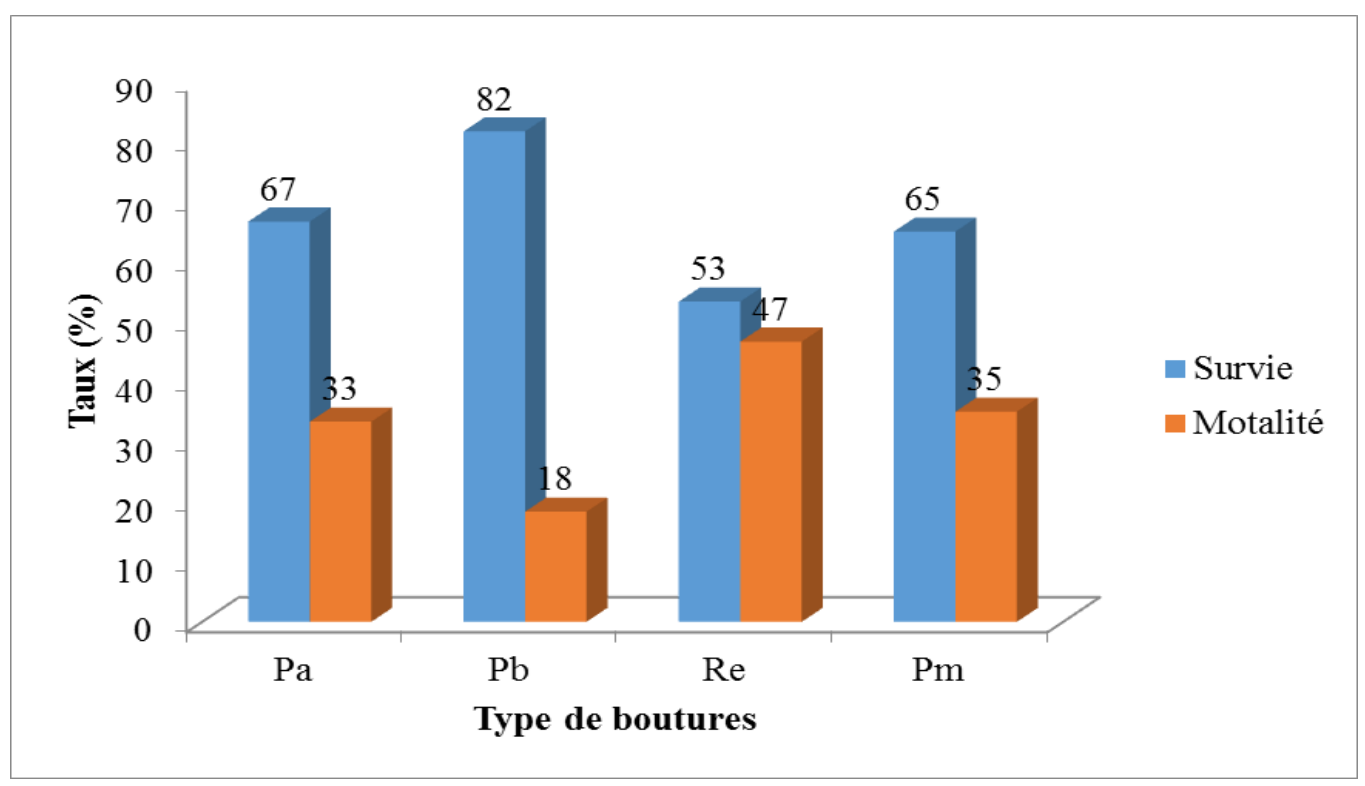

$\mathrm{Pa}=\mathrm{Partie}$ apicale, $\mathrm{Pm}=$ Partie médiane, $\mathrm{Pb}=$ Partie basale et $\mathrm{Re}=$ Raquette entière.

Figure 3: Etat de survie des plants en fonction du type de bouture. 


\section{DISCUSSION}

\section{Capacité de reprise des boutures}

La multiplication de l'espèce Opuntia ficus-indica par bouturage des raquettes donne des meilleurs résultats en terme de capacité de reprise de la plante au niveau des boutures, partie basale $(\mathrm{Pb})$ et apicale $(\mathrm{Pa})$ par rapport à la bouture, raquette entière. Ceci pourrait s'expliquer par le fait que les besoins physiologiques de base de la raquette entière sont d'au moins deux (2) fois supérieurs à ceux des deux (2) premières en biomasse à entretenir avant le développement du système radiculaire. La formation de ces racines a lieu quinze (15) jours après la plantation des raquettes (Araba et al., 2000 ; Mulas et Mulas, 2004). La légère baisse de la capacité de reprise des boutures observée entre le $28^{\text {ème }}$ et $35^{\text {ème }}$ jour après leur mise en pots serait liée à l'excès d'eau dû au pic de pluies généralement caractéristique du mois d'août, facteur non compatible avec le caractère xérophyte de l'espèce. La faible capacité de reprise observée au niveau de la bouture, partie médiane (Pm) semble liée à la double cicatrisation que celle-ci doit assurer avant le développement de son système radiculaire. Un autre facteur à incriminer serait le jeune âge des raquettes récoltées qui était de trois (03) mois contre l'âge de 3 à 5 ans recommandé (HCDS, 2002). Toutefois, en matière de bouturage, les boutures ne reproduisent à l'identique la plante-mère sélectionnée que si elles sont prélevées sur du matériel végétal jeune (Bellefontaine et al., 2015).

\section{Etat de survie des plants à la maturité des raquettes primaires émises}

Les meilleurs taux de survie obtenus respectivement au niveau des traitements, partie basale (82\%) et de celui constitué de la partie apicale $(67 \%)$ montrent un net avantage de gain en multiplication végétative de Opuntia ficus-indica par rapport à l'utilisation de la raquette entière (53\%), soit $20,5 \%$ de plus. Le taux de survie des plants issus de la bouture, partie médiane $(\mathrm{Pm})$, étant également supérieur à celui de la bouture, raquette entière (65\%) confirme l'intérêt de développer la technique de multiplication végétative de l'espèce par le découpage de la raquette en 2 ou 3 fragments. En effet, elle permet d'obtenir, dans les meilleurs délais, des quantités importantes des plants pour la mise en place d'un programme de production intensive de fourrage de l'espèce et de sa valorisation dans l'alimentation animale. Aussi, selon Bellefontaine et al. (2015), les plants issus de la multiplication végétative, en plus de leur mise en place à faible coût, assurent par leur enracinement et par leur feuillage, très tôt une protection des sols contre l'érosion, le vent et les tempêtes.

\section{Etat de développement végétatif des raquettes émises}

Au niveau des dimensions des deux (2) types de raquette, il est noté une faible variabilité entre les traitements par type de raquette, d'où l'absence de différence significative observée. Ceci expliquerait le fait que la technique de multiplication végétative utilisée permet de produire des plants à l'identique de la plante de laquelle les prélèvements d'organes ont été effectués (Bellefontaine et al., 2015). En effet, les raquettes ont été récoltées des plantes issues du micro plants produits en laboratoire entretenus en pépinière. La longueur moyenne de la raquette primaire variant de 19,05 à $22,02 \mathrm{~cm}$ avec une largeur moyenne comprise entre 6,73 et $13,37 \mathrm{~cm}$ sont inférieures aux valeurs observées par Chaouche et al. (2008) sur des raquettes des vieilles plantations de la même espèce en milieu steppique accusant une longueur moyenne de 35,71-35,76 cm et une largeur de $18,68-19,65 \mathrm{~cm}$ avec un rapport Largeur/Longueur de 0,52 à 0,55.

\section{Conclusion}

L'évaluation de la multiplication végétative de l'espèce Opuntia ficus-indica a montré qu'elle est une espèce fourragère prometteuse pour sa possibilité de multiplication des plants en grande quantité sous conditions pluviales en zone nordsoudanienne du Burkina Faso. Les résultats obtenus indiquent que la multiplication par la section de la raquette en deux parties serait mieux que celle consistant en la section de la 
raquette en trois parties. En perspectives, il serait souhaitable de poursuivre les investigations pour déterminer le mode cultural le plus adapté pour la culture de l'espèce en plein champ, tout en prenant en compte l'aspect phytopathologie et de conditions de son insertion dans les systèmes agropastoraux locaux. Aussi, des essais d'alimentation des bovins et des petits ruminants à base de raquettes produites s'imposent pour mieux déterminer les itinéraires techniques appropriés pour sa valorisation en alimentation animale dans les élevages périurbains et ruraux.

\section{CONTRIBUTIONS DES AUTEURS,}

MFO: Elaboration du protocole de recherche, traitement et analyse des données; CATG: Appui scientifique par la formation et en méthodologie; VB: Supervision scientifique de l'activité; HOS: Appui méthodologique et scientifique au cours de l'essai; DK: Appui en moyens matériels et financiers nécessaires à la conduite de l'essai, et appui technique; DKi: Mise en œuvre du protocole de recherche; AC: Appui technique à la mise en place et au suivi de l'activité ; MD: Appui technique à la conduite de l'essai.

\section{REMERCIEMENTS}

Ce travail a été réalisé dans le cadre du Projet Renforcement de l'Elevage Laitier au Burkina Faso, BRA/04/044S455 sous financement de la République Fédérative du Brésil et du Gouvernement du Burkina Faso à qui nous exprimons nos sincères remerciements et nos sentiments de profonde gratitude.

\section{REFERENCES}

Araba A, El Aich A, Sarti B, Belbahri L, Boubkraoui A, Ait Hammou A. 2000. Valorisation du figuier de Barbarie en élevage. Bulletin mensuel d'information et de liaison du PNTA. Transfert de technologie en Agriculture $\mathrm{n}^{\circ}$ 68, mai 2000, 9 p.

Arba M. 2009. Le cactus opuntia, une espèce fruitière et fourragère pour une agriculture durable au Maroc.
Symposium international «Agriculture durable en région Méditerranéenne (AGDUMED) », Rabat, Maroc, 14-16 mai $2009: 215-223$.

Bellefontaine R, Meunier Q, Ichaou A, Le Bouler H. 2015. « Multiplication végétative à faible coût au profit des paysans et éleveurs des zones tropicales et méditerranéennes », VertigO - la revue électronique en sciences de l'environnement [En ligne], Regards / Terrain, 2015, mis en ligne le 05 octobre 2015, consulté le 29 août 2016. URL : http://vertigo.revues.org/16516.

Bonogo WV. 2005. Phénologie, productivité, composition chimique et exploitation pastorale de Afzelia africana Smith ex Pers. et Pterocarpus erinaceus Poir. dans le Parc National KABORE Tambi (PNKT). Mémoire de fin d'études Institut du Développement Rural / Université Polytechnique de BoboDioulasso, $83 \mathrm{p}+$ annexes.

Chaouche TZ, Abdul-Hussain MS. 2008. Contribution à l'étude de l'Opuntia et perspectives d'amélioration, dans le milieu steppique. Agricultura, (1-2) : 6566.

CMAP (Centre National de Multiplication des Animaux Performants). 2015. Mémorandum sur la mission de la délégation brésilienne au Burkina Faso dans le cadre du «projet de renforcement de l'élevage laitier au Burkina Faso » 16 au 29 aout 2015. Ministère des ressources animales/Centre national de multiplication des animaux performants, $11 \mathrm{p}$.

Dembélé Y. 2010. Cartographie des Zones Socio-rurales. Burkina Faso. Un outil d'aide à la Planification pour la Gestion de l'Eau en Agriculture. FAO, 68 p.

EMBRAPA (Entreprise Brésilienne de Recherche Agro-pastorale). 2014. Tabelas nordestinas de composiçao de alimentos para bovinos leiteiros. Ministerio de Agricultura, Pecueria Abastecimento/Empresa Brasileira de Pesquisa Agropecuaria, Brasilia, 184 p ; 
HCDS. 2002. Opuntia ficus indica. Guide agropastoralisme. Notice bibliographique sur quelques plantes fourragères et pastorales, $3 p$

Kabore-Zoungrana CY, Dira B, Adandedjan C, Sawadogo S. 2008. Valeur nutritive de Balanites aegyptiaca pour l'alimentation des ruminants. Livestock Research for Rural Development, 20(4).

Klein HD, Rippstein G, Hugguenin J, Toutain B, Guerin H, Louppe D. 2014. Les cultures furragères. Centre technique de coopération agricole et rurale (CTA), Presses agronomiques de Gembloux. Belgique. $257 \mathrm{p}$.

MRA/DGPSE (Ministère des ressources animales/Direction générale des politiques et des statistiques en élevage). 2013. Annuaire des statistiques du secteur Elevage 2012, 152 p.

Mulas M, Mulas G. 2004. Potentialités d'utilisation stratégique des plantes des genres Atriplex et Opuntia dans la lutte contre la désertification, février 2004, $112 \mathrm{p}$.

Neffar S, Beddiar A, Rediel N, Boulkheloua J. 2011. Effets de l'âge des plantations de figuier de Barbarie (Opuntia ficus indica $f$ inermis) sur les propriétés du sol et la végétation à Tébessa (zone semi-aride de l'est algérien). Revue Internationale d'Ecologie Méditerranéenne, 37(1).
Neffar S, 2012. Etude de l'effet de l'âge des plantations de figuier de Barbarie (Opuntia ficus indica L. Miller) sur la variation des ressources naturelles (sol et végétation) des steppes lgériennes de l'Est. Cas de Souk- ahras et Tébessa. Thèse de Doctorat en biologie végétale. Université de Badji Mokctar Annaba. Republique Algérienne Démocratique et Populaire, $154 \mathrm{p}$.

Obulbiga MF, Bougouma V, Sanon HH, Gnanda I. 2014. Production à double objectif d'Andropogon gayanus Kunth en culture pluviale au Burkina Faso. Agronomie Africaine, 26(3) : 1 - 10.

Obulbiga MF, Kaboré-Zoungrana CY. 2007. Influence de la fumure azotée et du rythme d'exploitation sur la matière sèche et la valeur alimentaire de Andropogon gayanus Kunth au Burkina Faso. Tropicultura, 25(3): 161-167.

Sanon HO. 2007. The Importance of Some Sahelian Browse Species as Feed for Goats. Doctoral thesis. Swedish University of Agricultural Sciences Uppsala, 69 p.

Toutain B, Klein HD, Lhoste P, Duteurtre G. 2009. Histoire et avenir des cultures fourragères en Afrique tropicale. Fourrages, 2009: 511-523. 